\title{
Vacuum Brans-Dicke theory in the Jordan and Einstein frames: can they be distinguished by lensing?
}

\author{
R.N. Izmailov ${ }^{*}$ and R.Kh. Karimov \\ Zel'dovich International Center for Astrophysics, Bashkir State Pedagogical University, \\ 3A, October Revolution Street, Ufa 450008, RB, Russia \\ A.A. Potapov \\ Department of Physics \& Astronomy, Bashkir State University, \\ 49, Lenin street, Sterlitamak 453103, RB, Russia \\ K.K. Nand $\$$ \\ Zel'dovich International Center for Astrophysics, Bashkir State Pedagogical University, \\ 3A, October Revolution Street, Ufa 450008, RB, Russia and \\ High Energy Cosmic Ray Research Center, University of North Bengal, Darjeeling 734 013, WB, India
}

\begin{abstract}
Vacuum Brans-Dicke theory can be self-consistently described in two frames, the Jordan frame $(\mathrm{JF})$ and the conformally rescaled Einstein frame $(\mathrm{EF})$, the transformations providing an easy passage from one frame to the other at the level of actions and solutions. Despite this, the conformal frames are inequivalent describing different geometries. It is shown that the predictions of the weak field lensing (WFL) observables in the EF are different from those recently obtained in the JF for the vacuum Brans-Dicke class 1 solution. The value of the Brans-Dicke coupling parameter $\omega$ from the Cassini spacecraft experiment reveals the degree of accuracy needed to experimentally distinguish the WFL measurements including the total magnification factor in the two frames.
\end{abstract}

\section{INTRODUCTION}

Brans-Dicke (BD) theory [1-3], which describes gravitation through a spacetime metric $\left(g_{\mu \nu}\right)$, a massless scalar field $(\varphi)$ and a constant coupling parameter $\omega$, is a Machian competitor of Einstein's general relativity (GR). The theory continues to receive widespread attention since it is consistent with the solar and cosmological observations [4-12]. The vacuum BD theory that we shall consider here remarkably arises also in the low energy limit of heterotic string theory in 4-dimensions that predicts a model independent value $\omega=-1[13,14]$. The 1-parameter group of conformal symmetry of vacuum BD action discovered by Faraoni [7] can shift this value to any desired higher value of $\omega$ required by solar system observations.

A generic aspect of any scalar-tensor theory is that two frames are available for its description. One frame is the so-called Jordan frame (JF) in which the BD field equations were originally written and the scalar field $\varphi$ plays the role of a spin-0 component of gravity introduced to accommodate Mach's principle. The other is the conformally rescaled Einstein frame (EF) in which the transformed scalar field $\phi$ plays the role of material source stresses in the GR field equations. The curvature and geodesic properties are different in the two frames. Because of this, there have been a long standing debate

\footnotetext{
*Electronic address: izmailov.ramil@gmail.com

${ }^{\dagger}$ Electronic address: karimov_ramis_92@mail.ru

${ }^{\ddagger}$ Electronic address: a.a.potapov@strbsu.ru

$\S$ Electronic address: kamalnandi1952@yahoo.co.in
}

as to which of the two frames, JF and EF, should be considered physical. ${ }^{1}$

Depending on the attitude to this question, physicists are divided roughly into six groups. Some authors: (1) neglect the issue, (2) think that the two frames are physically equivalent, (3) consider them physically nonequivalent but do not provide supporting arguments, (4) regard only JF as physical but EF can be used for mathematical convenience, (5) regard only EF as physical, (6) belong to two or more of the above categories! For a detailed account, see the excellent review [9]. There are also arguments claiming that all physical observables are conformal frame invariants [15]. Some works in cosmology do show that it is indeed the case $[16,17]$. In this situation, we think that it is imperative to go beyond theoretical arguments favoring one position or the other but analyze tangible experimental predictions in the two frames. There exist very few works in this direction. One study involves the response of a gravitational wave detector to scalar waves, but the distinctive feature of a longitudinal mode, to be present in EF and absent in JF, seems unlikely to be observed in the near future [18].

In this paper, we consider the more pragmatic case of weak field lensing (WFL) in the EF and compare them

\footnotetext{
${ }^{1}$ A physical frame is the one in which test particles have constant masses and move along the geodesics in that frame. Vacuum $\mathrm{BD}$ theory in $\mathrm{JF}\left(T_{\mu \nu}^{(\text {matter })}=0\right)$ avoids anomalous coupling to matter in the EF. Then the EF solution is just the scalar field solution of GR corresponding to the vacuum solution in JF [29]. In this sense, both the frames are physical but observable predictions can still differ, which is the object of this paper.
} 
with those in the vacuum BD theory in the JF recently obtained by Gao et al [19]. Even though the GR theory in the EF has nothing to do with the Machian coupling parameter $\omega$, we show that the EF WFL observables can still be expressed as functions of $\omega$ making them amenable to the desired comparison. We shall then translate the value of $\omega$ obtained from the Cassini spacecraft experiment [20] into an accuracy level needed to distinguish the two frames.

In Sec.2, we state the solutions in JF and EF with the relation connecting the respective parameters. In Sec.3, we calculate the WFL observables for SgrA* and evaluate in Sec.4 the accuracies needed to observationally distinguish the two frames. Sec.5 discusses a useful WFL observable, the total magnification. Sec. 6 concludes the paper. We take $G=1, c=1$ unless specifically restored.

\section{JF AND EF}

(i) The vacuum BD action in the JF is [1]

$$
S_{\mathrm{JF}}=\frac{1}{16 \pi} \int d^{4} x(-g)^{\frac{1}{2}}\left[\varphi \mathbf{R}+\varphi^{-1} \omega g^{\mu \nu} \varphi_{, \mu} \varphi_{, \nu}\right] .
$$

The field equations are

$$
\begin{aligned}
\square^{2} \varphi & =0 \\
\mathbf{R}_{\mu \nu}-\frac{1}{2} g_{\mu \nu} \mathbf{R}= & -\frac{\omega}{\varphi^{2}}\left[\varphi_{, \mu} \varphi_{, \nu}-\frac{1}{2} g_{\mu \nu} \varphi_{, \sigma} \varphi^{, \sigma}\right] \\
& -\frac{1}{\varphi}\left[\varphi_{; \mu} \varphi_{; \nu}-g_{\mu \nu} \square^{2} \varphi\right]
\end{aligned}
$$

where $\square^{2} \equiv\left(\varphi^{; \rho}\right)_{; \rho}$ and $\omega$ is a dimensionless constant coupling parameter. The general solution, in isotropic coordinates $(t, r, \theta, \varphi)$, is given by

$$
\begin{aligned}
d \tau^{2}=g_{\mu \nu} d x^{\mu} d x^{\nu}= & -e^{2 \alpha(r)} d t^{2}+e^{2 \beta(r)} d r^{2} \\
& +e^{2 \nu(r)} r^{2}\left(d \theta^{2}+\sin ^{2} \theta d \psi^{2}\right)
\end{aligned}
$$

Brans class I solution $[2,3]$ correspond to the gauge $\beta-$ $\nu=0$ and is given by

$$
\begin{aligned}
e^{\alpha(r)} & =e^{\alpha_{0}}\left[\frac{1-B / r}{1+B / r}\right]^{\frac{1}{\lambda}}, \\
e^{\beta(r)} & =e^{\beta_{0}}[1+B / r]^{2}\left[\frac{1-B / r}{1+B / r}\right]^{\frac{\lambda-C-1}{\lambda}}, \\
\varphi(r) & =\varphi_{0}\left[\frac{1-B / r}{1+B / r}\right]^{\frac{C}{\lambda}}, \\
\lambda^{2} & \equiv(C+1)^{2}-C\left(1-\frac{\omega C}{2}\right)>0,
\end{aligned}
$$

where $\alpha_{0}, \beta_{0}, B, C$, and $\varphi_{0}$ are constants. The constants $\alpha_{0}$ and $\beta_{0}$ are determined by asymptotic flatness condition as $\alpha_{0}=\beta_{0}=0$. (ii) Under the conformal transformation (often called Dicke transformations [4])

$$
\widetilde{g}_{\mu \nu}=p g_{\mu \nu}, \quad p=\frac{1}{16 \pi} \varphi
$$

and a redefinition of the BD scalar

$$
d \phi=\left(\frac{\omega+3 / 2}{\kappa}\right)^{1 / 2} \frac{d \varphi}{\varphi}
$$

the action $(1)$ in the $\operatorname{EF}\left(\widetilde{g}_{\mu \nu}, \phi\right)$ becomes

$$
S_{\mathrm{EF}}=\int d^{4} x(-\widetilde{g})^{1 / 2}\left[\widetilde{\mathbf{R}}+\kappa \widetilde{g}^{\mu \nu} \phi_{, \mu} \phi_{, \nu}\right]
$$

leading to the field equations

$$
\begin{aligned}
\widetilde{\mathbf{R}}_{\mu \nu} & =-\kappa \phi_{, \mu} \phi_{, \nu} \\
\square^{2} \phi & =0 .
\end{aligned}
$$

The arbitrary constant $\kappa$ can have any sign \pm 1 but we chose $\kappa=+1$ in order to ensure that the stress of the source scalar field $\phi$ satisfies the energy conditions. The solutions of Eqs. (12) and (13) can be obtained, using the transformations () and (). Redefining $B=\frac{m}{2}$, we then get what is called the Buchdahl solution $[21,22]^{2}$

$$
\begin{aligned}
d \tau^{2}= & -\left(1+\frac{m}{2 r}\right)^{-2 \gamma}\left(1-\frac{m}{2 r}\right)^{2 \gamma} d t^{2} \\
& +\left(1+\frac{m}{2 r}\right)^{2(1+\gamma)}\left(1-\frac{m}{2 r}\right)^{2(1-\gamma)} \times \\
\phi(r)= & \sqrt{\left(\omega+\frac{3}{2}\right)\left(\frac{C^{2}}{\lambda^{2}}\right)} \ln \left[\frac{1-\frac{m}{2 r}}{1+\frac{m}{2 r}}\right] . \\
\gamma= & \frac{1}{\lambda}\left(1+\frac{C}{2}\right) .
\end{aligned}
$$

With this $\gamma$, and using (8), the scalar field can be reexpressed as

$$
\phi(r)=\sqrt{2\left(1-\gamma^{2}\right)} \ln \left[\frac{1-\frac{m}{2 r}}{1+\frac{m}{2 r}}\right] .
$$

The solution $(14,17)$ is now completely characterized by a single free parameter $\gamma$, a constant of integration of GR equations $(12,13)$ that no longer has anything to do with the Machian parameter $\omega$.

However, in the limit $\omega \rightarrow \infty$, one should recover GR from Brans-Dicke theory ${ }^{3}$. But the Buchdahl solution

\footnotetext{
${ }^{2}$ It is also called Fisher solution [23] or Bergman-Leipnik solution [24] caused by normal matter field but the solutions was expressed in a non-transparent form. We are indebted to Prof. K.A. Bronnikov for pointing it out.

3 The limit $\omega \rightarrow \infty$ is not as simple as it looks. The passage to GR in that limit is said to be anomalous and is still being discussed (see e.g., [25-29]), but we disregard these issues here. Most recently, Faraoni and Côté [29] have shown that the symmetry is preserved even in the limit $\omega \rightarrow \infty$.
} 
self-consistently satisfies the EF equations $(12,13)$, where the metric (14) and the scalar field $\phi$ in Eq.(17) have no $\omega$ dependence and the limit $\omega \rightarrow \infty$ has no meaning. However, the intermediate expression (15) for $\phi$ does contain $\omega$ and in that case, one might hope to talk of the limit $\omega \rightarrow \infty$. For this limit in EF to be meaningful, the WFL observables from both the metrics (4) and (14) should exactly coincide in this limit. To show that this is indeed the case, one then has to use the previous incarnation of $\gamma$ connecting it to $\omega$.

Using the weak field expression for

$$
C=-\frac{1}{2+\omega}
$$

in $\lambda$ in (8) and $\gamma$ in (16), it follows that

$$
\gamma=\lambda=\sqrt{\frac{3+2 \omega}{4+2 \omega}}
$$

which shows $\gamma \rightarrow 1$ as $\omega \rightarrow \infty$. The expansion of JF metric component.(5) yields

$$
e^{2 \alpha(r)} \simeq 1-\frac{4 B}{\lambda r} \simeq 1-\frac{2 M}{r}
$$

from which one obtains the Schwarzschild mass $M_{\text {Sch }}^{\mathrm{JF}}=$ $\frac{2 B}{\lambda}$, and similarly from the EF metric component in (14), $M_{\mathrm{Sch}}^{\mathrm{EF}}=m \gamma$. The ADM mass for JF metric (4) is $M_{\mathrm{ADM}}^{\mathrm{JF}}=\frac{2 B}{\lambda}(C+1)=M_{\mathrm{Sch}}^{\mathrm{JF}}(C+1)$. Similarly, for $\mathrm{EF}$ metric (14), the mass is $M_{\mathrm{ADM}}^{\mathrm{EF}}=m \gamma=M_{\mathrm{Sch}}^{\mathrm{EF}}$ and we already see that the gravitating masses are different in JF and EF. Thus the frames are qualitatively distinguishable already showing the inequivalence of the JF and EF though all the masses coincide at $\omega \rightarrow \infty$. However, we want to know how the experimental observables quantitatively differ between them for finite $\omega$, for which we turn to WFL observables.

\section{WFL OBSERVABLES}

Gao et al [19] have extended Keeton-Petters method [30] up to 4th order, which essentially are the PPN coefficients of the light deflection angle (for some useful recent applications of the method, see $[31,32])$. For the vacuum $\mathrm{BD}$ solution in the JF, the coefficients are (we quote Gao's coefficients up to third order without any loss of rigor for our argument):

$$
\begin{aligned}
A_{1}^{\mathrm{JF}} & =\frac{6+4 \omega}{2+\omega}, \\
A_{2}^{\mathrm{JF}} & =\frac{\pi\left(30 \omega^{2}+89 \omega+66\right)}{8(2+\omega)^{2}}, \\
A_{3}^{\mathrm{JF}} & =\frac{2(3+2 \omega)^{2} \pi\left(30 \omega^{2}+89 \omega+66\right)}{8(2+\omega)^{2}} .
\end{aligned}
$$

For the Buchdahl solution of GR, the PPN coefficients are expressed in terms of $\gamma$. When expressed in terms of $\omega$ up to third order, they yield

$$
\begin{aligned}
& A_{1}^{\mathrm{EF}}=4, \\
& A_{2}^{\mathrm{EF}}=\pi\left(4-\frac{1}{4 \gamma^{2}}\right)=\frac{\pi(22+15 \omega)}{6+4 \omega}, \\
& A_{3}^{\mathrm{EF}}=16\left(3-\frac{1}{3 \gamma^{2}}\right)=\frac{368+256 \omega}{9+6 \omega} .
\end{aligned}
$$

They are evidently not the same expressions derived by of Gao et al [19] directly from the original vacuum BransDicke theory in the JF. However, as $\omega \rightarrow \infty$, we retrieve the vacuum $G R$ values

$$
A_{1}=4, \quad A_{2}=\frac{15 \pi}{4}, \quad A_{3}=\frac{128}{3},
$$

and similarly following the method of Gao et al [19], we have verified in the $\mathrm{EF}$ that $A_{4}=\frac{3465 \pi}{64}$ in that limit. These are exactly the same values obtained also from Eqs.() in the same $\omega \rightarrow \infty$ limit. The arguments and calculations above illuminate the fundamental difference between the lensing observables of Brans-Dicke theory and Einstein's theory except in the limit $\omega \rightarrow \infty$.

Since, after all, $\omega$ is not infinite but very large, it would be curious to see how much accuracy in measurement would be required to distinguish the WFL observables between JF and EF. A value of $\omega$ follows from the Cassini spacecraft experiment by Bertotti et al [20], which we shal use. They measured the round trip time delay $\Delta t$ of light between the ground antenna and the spacecraft, at distances $r_{1}$ and $r_{2}$ respectively from the Sun with mass $M_{\odot}$ using the model independent Robertson Coefficient $\gamma^{\mathrm{RC}}$ of the centrally symmetric gravity field [33] [Note: $\gamma^{\mathrm{RC}}$ has nothing to do with the EF $\gamma$ in Eq.(16)]:

$$
\Delta t=2\left(1+\gamma^{\mathrm{RC}}\right) \frac{G M_{\odot}}{c^{3}} \ln \left(\frac{4 r_{1} r_{2}}{b^{2}}\right),
$$

where $\left(r_{1}, r_{2}\right)<<b$, the impact parameter. The value of $\gamma^{\mathrm{RC}}$ is 0 in Newtonian gravity and 1 in GR. The result of the Cassini spacecraft experiment is $\gamma^{\mathrm{RC}}=$ $1+(2.1 \pm 2.3) \times 10^{-5}[20]$. In the BD theory, one has $\gamma^{\mathrm{RC}}=\frac{1+\omega}{2+\omega}$, which leads to a value $|\omega| \sim 50,000$.

We consider the WFL observables to be the image position $\theta$ (scaled by the Einstein angle $\mathfrak{v}_{E}$ ) and total magnification $\mu_{\text {tot }}$. Consider the expansion

$$
\theta=\theta_{0}+\theta_{1} \varepsilon+\theta_{2} \varepsilon^{2}+\ldots,
$$

where, for an arbitrary gravitating mass $M$, and the small expansion parameter $\varepsilon$,

$$
\begin{aligned}
\varepsilon & =\frac{\theta_{\bullet}}{\mathfrak{v}_{E}}, \theta_{\bullet}=\tan ^{-1}\left(\frac{m_{\bullet}}{d_{O L}}\right), \\
m_{\bullet} & =\frac{G M}{c^{2}}, \mathfrak{v}_{E}=\left(\frac{4 M d_{L S}}{d_{O L} d_{O S}}\right)^{1 / 2},
\end{aligned}
$$

where $d_{O L}$ is the observer-lens distance, $d_{O S}$ is the observer-source distance and $d_{L S}$ is the lens-source distance. We shall consider the known black hole SgrA* as 
the lens and for its mass, the correction $\theta_{1} \varepsilon$ is too small compared to the zeroth order term $\theta_{0}$ in both frames as we will soon see. In the JF, the coefficients are [19] (considering only the positive parity image):

$$
\begin{aligned}
& \theta_{0}^{\mathrm{JF}}=\frac{1}{2}\left[\beta+\left(\sqrt{\beta^{2}+4\left(\frac{3+2 \omega}{4+2 \omega}\right)}\right)\right], \\
& \theta_{1}^{\mathrm{JF}}=\frac{\pi\left(30 \omega^{2}+89 \omega+66\right)}{16(\omega+2)\left[2 \theta_{0}^{2}(2+\omega)+3+2 \omega\right]},
\end{aligned}
$$

Similarly, in the EF, we find that

$$
\begin{aligned}
\theta_{0}^{\mathrm{EF}} & =\frac{1}{2}\left[\beta \pm\left(\sqrt{\beta^{2}+A_{1}^{\mathrm{EF}}}\right)\right] \\
& =\frac{1}{2}\left[\beta+\left(\sqrt{\beta^{2}+4}\right)\right] \\
\theta_{1}^{\mathrm{EF}} & =\frac{A_{2}^{\mathrm{EF}}}{A_{1}^{\mathrm{EF}}+4\left(\theta_{0}^{\mathrm{EF}}\right)^{2}} \\
& =\frac{\pi(22+15 \omega)}{(6+4 \omega)\left[4+\left(\beta+\sqrt{\beta^{2}+4}\right)^{2}\right]},
\end{aligned}
$$

Technically, it is difficult to measure individual magnifications of images, so we consider the total magnification, which are [19]

$$
\begin{aligned}
\mu_{0, \text { tot }}^{\mathrm{JF}} & =\frac{A_{1}^{\mathrm{JF}}+2 \beta^{2}}{2 \beta \sqrt{A_{1}^{\mathrm{JF}}+\beta^{2}}}=\frac{\beta^{2}(\omega+2)+3+2 \omega}{\beta(\omega+2) n} \\
\mu_{1, \text { tot }}^{\mathrm{JF}} & =-\frac{A_{2}^{\mathrm{JF}}}{2\left(A_{1}^{\mathrm{JF}}+\beta^{2}\right)^{3 / 2}} \\
& =-\frac{\pi(3+2 \omega)(15 \omega+22) n}{16\left[\beta^{2}(2+\omega)+4 \omega+6\right]^{2}} \\
n & =\sqrt{\beta^{2}-\frac{2}{2+\omega}+4} .
\end{aligned}
$$

Similarly, in the EF,

$$
\begin{aligned}
& \mu_{0, \text { tot }}^{\mathrm{EF}}=\frac{A_{1}^{\mathrm{EF}}+2 \beta^{2}}{2 \beta \sqrt{A_{1}^{\mathrm{EF}}+\beta^{2}}}=\frac{2+\beta^{2}}{\beta \sqrt{4+\beta^{2}}} \\
& \left.\mu_{1, \text { tot }}^{\mathrm{EF}}=-\frac{A_{2}^{\mathrm{EF}}}{2\left(A_{1}^{\mathrm{EF}}+\beta^{2}\right)^{3 / 2}}=-\frac{\pi(22+15 \omega)}{2(6+4 \omega)\left(4+\beta^{2}\right)^{3 / 2}}\right)
\end{aligned}
$$

where we have used $A_{1}^{\mathrm{EF}}=4$.

\section{ACCURACIES NEEDED}

To get a flavor of the accuracy needed, we take, for illustration, $\beta=0.1$ and following the relevant values for the lens $\operatorname{SgrA}^{*}[30]$ with $d_{L S}=10$ parsec, we take the small expansion parameter to be $\varepsilon=1.3 \times 10^{-4}$, the
Einstein angle $\mathfrak{v}_{E}=0.068$ arcsec, and from the Cassini spacecraft experiment, $\omega=50000$ [20]. Restoring the dimensions by multiplying both sides of the series by $\mathfrak{v}_{E}$, we get

$$
\begin{aligned}
\theta_{0}^{\mathrm{JF}} \mathfrak{v}_{E} & =71484.94 \mu \operatorname{arcsec} \\
\theta_{1}^{\mathrm{JF}} \varepsilon \mathfrak{v}_{E} & =12.3678 \mu \operatorname{arcsec} \\
\Rightarrow \theta_{\text {tot }}^{\mathrm{JF}} & =\theta_{0}^{\mathrm{JF}} \mathfrak{v}_{E}+\theta_{1}^{\mathrm{JF}} \varepsilon \mathfrak{v}_{E}=71497.314 \mu \operatorname{arcsec}(40) \\
\theta_{0}^{\mathrm{EF}} \mathfrak{v}_{E} & =71484.6 \mu \operatorname{arcsec}, \\
\theta_{1}^{\mathrm{EF}} \varepsilon_{E} & =12.3678 \mu \operatorname{arcsec} \\
\Rightarrow \theta_{\text {tot }}^{\mathrm{EF}} & =\theta_{0}^{\mathrm{EF}} \mathfrak{v}_{E}+\theta_{1}^{\mathrm{EF}} \varepsilon \mathfrak{v}_{E}=71496.975 \mu \operatorname{arcse}(42)
\end{aligned}
$$

The qualitative conclusion is that the image angular positions are closer to the optical axis $O L$ in the EF than in the JF. Evidently, the first order corrections are too small in both frames compared to the zeroth order, $\theta_{1}^{\mathrm{JF}, \mathrm{EF}} \varepsilon \mathfrak{v}_{E}<<\theta_{0}^{\mathrm{JF}, \mathrm{EF}} \mathfrak{v}_{E}$ and higher order terms would be even smaller, hence ignored. Looking at the total angular position, $\theta_{\text {tot }}^{\mathrm{JF}}$ and $\theta_{\text {tot }}^{\mathrm{JF}}$, it is clear that future astrometric missions would need to attain an angular measurement accuracy better that $0.3 \mu$ arcsec to observationally distinguish between the two frames. This is certainly a challenging task but not impossible in the future. Likewise

$$
\begin{aligned}
\mu_{0, \text { tot }}^{\mathrm{JF}} & =10.0374, \mu_{1, \text { tot }}^{\mathrm{JF}}=-0.733554 \\
\mu_{\mathrm{tot}}^{\mathrm{JF}} & =9.30386 \\
\mu_{0, \text { tot }}^{\mathrm{EF}} & =10.0375, \mu_{1, \text { tot }}^{\mathrm{EF}}=-0.733558, \\
\mu_{\mathrm{tot}}^{\mathrm{EF}} & =9.30390, \\
& \Rightarrow \mu_{\mathrm{tot}}^{\mathrm{JF}} / \mu_{\mathrm{tot}}^{\mathrm{EF}}=1-5 \times 10^{-6} .
\end{aligned}
$$

This shows that, qualitatively, the image magnification is more in the EF than in the JF. In practice, one measures the magnification factor [34], which seems a bit easier to observe, to which we devote the next section.

\section{MAGNIFICATION FACTOR}

We start with the leading order angular positions $\theta_{ \pm}$ of the images dropping the subscript 0:

$$
\theta_{ \pm}=\frac{1}{2}\left[\beta \pm\left(\sqrt{\beta^{2}+A_{1}}\right)\right]
$$

where $A_{1}$ could be giv $A_{1}^{\mathrm{JF}}$ or $A_{1}^{\mathrm{EF}}=4$ and the angles for both parity images $\theta_{ \pm}$and $\beta$ are as before scaled by the Einstein angle $\mathfrak{v}_{E}$. The polar width $\Delta \theta_{ \pm}$of the images change and its magnitude can be obtained by differentiating Eq.(63):

$$
\Delta \theta_{ \pm}=\left(\frac{1}{2}\right)\left[1 \pm \frac{\beta}{\sqrt{\beta^{2}+A_{1}}}\right] \Delta \beta .
$$

Since the angular width of the source $\Delta \beta \neq 0$, this result implies a distorted and elongated shape of the images that have been confirmed by observations. 
The ratio of the brighness of the individual images $\mu_{ \pm}$ to the unlensed brightness $\mu_{*}$ at the angular positions $\theta_{ \pm}$ is given by the individual magnifications

$$
\begin{aligned}
\frac{\mu_{ \pm}}{\mu_{*}} & =\left|\left(\frac{\theta_{ \pm}}{\beta}\right)\left(\frac{d \theta_{ \pm}}{d \beta}\right)\right| \\
& =\frac{1}{4}\left[\frac{\beta}{\sqrt{\beta^{2}+A_{1}}}+\frac{\sqrt{\beta^{2}+A_{1}}}{\beta} \pm 2\right] .
\end{aligned}
$$

We can draw a very interesting conclusion from here: Since $x+1 / x \geq 2$, we can conclude that, for $\beta<0$, the brightness $\left|\mu_{-}\right|>\mu_{+}$showing that the image $\theta_{-}$is brighter than the image $\theta_{+}$. However, the magnitudes of individual image brightness $\mu_{ \pm}$for any given source do not differ very greatly thus making the individual measurements difficult. In this case, another very useful quantity is the total magnification over the background $\mu_{*}$, called the magnification factor $\mathcal{F}$ defined by (see for details, Hartle [34])

$$
\begin{aligned}
\mathcal{F}^{\mathrm{JF}} & =\left.\frac{\mu_{\mathrm{tot}}}{\mu_{*}}\right|_{\mathrm{JF}}=\frac{\mu_{+}-\mu_{-}}{\mu_{*}}=\frac{1}{2}\left[\frac{\beta}{\sqrt{\beta^{2}+A_{1}}}+\frac{\sqrt{\beta^{2}+A_{1}}}{\beta}\right] \\
& =\frac{1}{2}\left[\frac{\beta}{\sqrt{\beta^{2}+4\left(\frac{3+2 \omega}{4+2 \omega}\right)}}+\frac{\sqrt{\beta^{2}+4\left(\frac{3+2 \omega}{4+2 \omega}\right)}}{\beta}\right],
\end{aligned}
$$

the ratio being always greater than unity. For GR, $A_{1}=$ 4 and so

$\mathcal{F}^{\mathrm{EF}}=\left.\frac{\mu_{\mathrm{tot}}}{\mu_{*}}\right|_{\mathrm{EF}}=\frac{\mu_{+}-\mu_{-}}{\mu_{*}}=\frac{1}{2}\left[\frac{\beta}{\sqrt{\beta^{2}+4}}+\frac{\sqrt{\beta^{2}+4}}{\beta}\right]$.

The best result is obtained when $\beta$ is small, that is, when the source is very close to the optical axis $O L$, the total magnification factor could be quite large that should be observable. Accordingly, we take $\beta=0.1$ and translate the limit on $\omega=50000$ from the Cassini spececraft experiment [20] into image magnification factor. Then we find that

$$
\frac{\mathcal{F}^{\mathrm{JF}}}{\mathcal{F}^{\mathrm{EF}}}=1-4.9 \times 10^{-6},
$$

which is expectedly almost the same as in Eq.(47). Recent measurements of gravitationally lensed quasars provide total magnifications with too wide error margins and the factor ratio is impossible to measure [35]. Measurements of image positions could probably be easier provided an accurary at the level of fractional $\mu$ arcsec is attained, challenging but not impossible.

\section{CONCLUSIONS}

The answer to the question posed in the title is yes. We considered the vacuum Brans class I solution (4-8) and its conformal transform to the Buchdahl solution $(14,17)$, both solutions being self consistent and physical as defined in the introduction. It is evident that these solutions together with their parent theories, vacuum BD and GR respectively, can very well exist independently of knowing each other. In this case, the constants $(C, \lambda, \omega)$ from the solutions (4-8) and $\gamma$ from $(14,17)$ are unconnected since $\omega$ has no meaning in the $\mathrm{EF}$. But if one believes that the matter scalar field $\phi$ in the $\mathrm{EF}$ still has a memory of its previous incarnation of 0 -component gravity in the JF, then the connection Eq.(16) has a meaning and using it, one can easily retrieve the JF metric from the EF metric and vice versa. However, nobody measures the metric but only the experimental observables, which we have chosen to be the WFL observables.

In general, reformulation of any scalar-tensor theory in a new conformal frame leads to anomalous coupling to matter making the reformulation a physically inequivalent theory and it is believed that experiments involving motion of massive timelike particles would reveal this dif(51ference [10]. We showed here that quantitatively different results appear for finite $\omega$ even when the anomalous coupling is absent.

Recent derivations by Gao et al [19] of the WFL obervables in the JF for $C(\omega)=-\frac{1}{2+\omega}$ have given us an opportunity to exactly compare their results with those obtained in the EF. Using the expression for $\gamma(\omega)$ of Eq.(19) in the EF observables, we argue that one cannot retrieve the values of WFL observables in the JF. As illustration, we chose the black hole $\mathrm{SgrA}^{*}$ as the lens and used the value $\omega=50000$ obtained in the Cassini spacecraft experiment [20] to show that angular measurement accuracy better that $0.3 \mu$ arcsec is required to observationally distinguish between the two frames. In the same way, the image magnification would differ in the two frames by a factor $\left(1-4.9 \times 10^{-6}\right)$. The accuracy needed in both cases is challenging but may not be impossible to attain in the future.

\section{Aknowledgements}

The reported study was funded by RFBR according to the research project No. 18-32-00377. 
[3] K.K. Nandi, A. Islam and J. Evans, Phys. Rev. D 55, 2497 (1997).

[4] G. Magnano and L.M. Sokolowski, Phys. Rev. D 50, 5039 (1994).

[5] P.J. Steinhardt and F.S. Accetta, Phys. Rev. Lett. 64, 2740 (1990).

[6] O. Bertolami and P.J. Martins, Phys. Rev. D 61, 064007 (2000).

[7] V. Faraoni, Phys. Lett. A 245, 26 (1998).

[8] A. Bhadra, K. Sarkar, D.P. Datta and K.K. Nandi, Mod. Phys. Lett. A 22, 367 (2007).

[9] L. Qiang, Y. Ma, M. Han and D. Yu, Phys. Rev. D 71, 061501 (2005).

[10] V. Faraoni, E. Gunzig and P. Nardone, Fund. Cosmic Phys. 20, 121 (1999).

[11] Y.M. Cho, Phys. Rev. Lett. 68, 3133 (1992).

[12] V. Faraoni, Phys. Rev. D 59, 084021 (1991).

[13] K.K. Nandi and Y.-Z. Zhang, Phys. Rev. D 70, 044040 (2004).

[14] S. Kar, Class. Quant. Grav. 16, 101 (1999).

[15] É.É. Flanagan, Class. Quant. Grav. 21, 3817 (2004).

[16] E.W. Kolb, D.S. Salopek and M.S. Turner, Phys. Rev. D 42, 3925 (1990).

[17] C. Armendaríz-Pícon, Phys. Rev. D 66, 064008 (2002).

[18] S. Bellucci, V. Faraoni and D. Babusci, Phys. Lett. A 282, 357 (2001).

[19] X. Gao, S. Song and J. Yang, Phys. Lett. B 795, 144 (2019).
[20] B. Bertotti, L. Iess and P. Tortora, Nature (London) 425, 374 (2003).

[21] H.A. Buchdahl, Phys. Rev. 115, 1325 (1959).

[22] K.K. Nandi, B. Bhattacharjee, S.M.K. Alam and J. Evans, Phys. Rev. D 57, 823 (1998).

[23] I.Z. Fisher, Zh. Eksp. Teor. Fiz. 18, 636 (1948).

[24] O. Bergman and R. Leipnik, Phys. Rev. 107, 1157 (1957).

[25] A. Bhadra and K. K. Nandi, Phys. Rev. D 64, 087501 (2001).

[26] C. Romero and A. Barros, Phys. Lett. A 173, 243 (1993).

[27] N. Banerjee and S. Sen, Phys. Rev. D 56,1334 (1997).

[28] V. Faraoni, Phys. Rev. D 59, 084021 (1999).

[29] V. Faraoni and J. Côté, Phys. Rev. D 99, 064013 (2019).

[30] C.R. Keeton and A.O. Petters, Phys. Rev. D 72, 104006 (2005).

[31] R.N. Izmailov, R. Kh. Karimov, E.R. Zhdanov and K.K. Nandi, Mon. Not. R. Astron. Soc. 483, 3754 (2019).

[32] R.F. Lukmanova, G.Y. Tuleganova, R.N. Izmailov and K.K. Nandi, Phys. Rev. D 97, 124027 (2018).

[33] S. Weinberg, Gravitation and Cosmology (Wiley, New York,1972).

[34] J.B. Hartle, Gravity: An Introduction to Einstein's General Relativity (Pearson Inc. San Francisco, 2003).

[35] C.A. Lemon, M.W. Auger, R.G. McMahon and F. Ostrovski, Mon. Not. R. Astron. Soc. 479, 5060 (2018). 